\title{
Learning for the next pandemic: the Wuhan experience of managing critically ill people
}

\author{
Bin Du and colleagues explore the Wuhan response to managing critically ill patients in a novel \\ disease pandemic, and lessons learnt for dealing with future pandemics
}

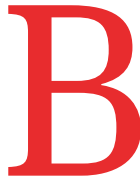

eing one of the countries most affected by natural disasters, China is well prepared to cope with mass casualty events. Having a centralised national health care system enables a more efficient mobilisation of resources and provision of external support, ${ }^{1}$ organised by the National Health Commission (NHC) and local healthcare authorities.

Although severe acute respiratory syndrome (SARS) affected only a few provinces and cities such as Beijing and Guangdong province in 2003, ${ }^{2}$ this experience triggered the National Emergency Plan for Public Health Emergencies, published in 2006. As a result, an infrastructure to deal with contagious diseases has been in place in most hospitals in China, including a fever clinic (with isolation rooms for observation and basic treatment) plus availability of personal protective equipment (PPE) and equipment for patient care. Severely ill patients with a confirmed contagious disease (such as influenza pneumonia) are admitted to

\section{KEY MESSAGES}

- China has a centralised national response system that can mobilise local and national healthcare resources quickly to cope with mass casualty events

- A local command centre is responsible for coordination and allocation of healthcare resources based on dynamic information

- Healthcare capacity, including critical care, may be expanded in a rapid manner to meet a patient surge, with quality of care maintained by imported teams of well trained specialty professionals

- A national emergency plan enables coordination and mobilisation of necessary resources during a pandemic, while early experience sharing should be organised in a systematic approach to provide consensus-based guidance on patient management local hospitals with relevant facilities and capabilities or are transferred to specialised hospitals at the discretion of the treating physicians. Some hospitals run infection control training programmes, and clinicians were required to obtain annual CME credits. However, such preparations had lapsed somewhat as no major outbreak had occurred since 2003. Nonetheless, this structure was not designed for a massive patient surge such as that experienced in Wuhan.

\section{Mobilising resources at short notice}

At the onset of the outbreak in Wuhan, the local healthcare authorities required all patients with suspected or laboratory confirmed covid-19 to be admitted to two specialised infectious diseases hospitals. As these hospitals mainly admit patients with active tuberculosis or viral hepatitis, they lacked sufficient critical care resources to cope with the numbers presenting. As per the emergency plan, the local healthcare authorities requested external assistance from tertiary hospitals in Wuhan, including infectious disease specialists, pulmonologists, critical care physicians, and nurses. Subsequently, 12 hospitals were designated to admit only covid-19 patients while other hospitals took non-covid emergencies and patients requiring longer term treatments such as dialysis and chemotherapy. All scheduled surgery was cancelled. Sixteen shelter hospitals were later opened to manage mild cases of covid19 and close contacts. ${ }^{3}$

As soon as it was recognised that local healthcare resources could not manage the patient surge, the NHC issued administrative orders to almost all provincial healthcare authorities for medical rescue teams, with specialties specified according to local needs. A command centre was established in Wuhan, comprising officials from both local and national healthcare authorities, and approximately 20 medical experts (including the author BD) from different specialties. The centre was responsible for assessing the patient surge, strains on the healthcare system, and resource allocation including workforce, equipment, drugs, consumables, and PPE. Information was gathered from the hospitals on a daily basis, including numbers of new admissions, inpatients, critically ill patients, ventilated patients, deaths, and discharges.

Between 24 January and 1 March 2020, 344 medical rescue teams comprising 42322 healthcare workers (11416 physicians, 28679 nurses) arrived in Hubei Province, of whom 38478 were deployed in Wuhan. ${ }^{4}$ As Wuhan is a large city of over 14 million people and a major transport hub, all could be accommodated in local hotels.

\section{Increasing critical care capacity}

As the intensive care facilities in the designated covid-19 hospitals were soon overwhelmed by large numbers of critically ill patients, many patients had to be managed on general wards, including some requiring non-invasive or even invasive mechanical ventilation. Under the direction of infection control specialists, wards were rapidly modified to admit covid-19 patients, and intensive care beds were created by co-locating monitors and ventilators. Non-intensive care staff worked under the supervision of critical care physicians and nurses. Extra medical equipment was purchased by central government and allocated to the hospitals by the command centre. In some hospitals, specialised teams of intensive care and anaesthesia staff were created to manage aerosol-generating procedures such as airway management, intubation, tracheostomy, and bronchoscopy. Later, these were expanded to other teams providing renal replacement therapy and extracorporeal membrane oxygenation.

\section{Scaling up critical care resources}

Before the pandemic struck, there were approximately 600 intensive care beds, 300 intensivists, and 1000 intensive care nurses in Wuhan serving a population over 14 million people. ${ }^{5}$ While the number of new intensive care beds created was not recorded, table 1 indicates the demand during the surge in case, albeit data are lacking for the onset.

The national experts participated in daily ward rounds to review the most critically ill patients. If the treating physicians 


\begin{tabular}{|c|c|c|c|c|}
\hline Date & Inpatients & Critically ill patients & Patients receiving NIV & Patients receiving IMV \\
\hline 12 February* & 3343 & 451 & 138 & 47 \\
\hline 19 February* & 5223 & 569 & 149 & 130 \\
\hline 26 February* & 5927 & 538 & 93 & 153 \\
\hline 4 March & 7366 & 592 & 86 & 182 \\
\hline 11 March & 6203 & 477 & 56 & 140 \\
\hline 17 March & 4062 & 344 & 28 & 144 \\
\hline 24 March & 2243 & 212 & 19 & 110 \\
\hline 31 March & 982 & 111 & 11 & 61 \\
\hline
\end{tabular}

considered that any patient was too severely ill to be managed on their wards, the patient would be transferred to other designated hospitals with appropriate facilities and capabilities, coordinated by the command centre. No patients were transferred to other cities, as national law prohibits long distance transfer of patients with contagious diseases. Furthermore, as Wuhan is well provisioned with hospital beds, the challenges lay more in remodelling hospitals and coping with healthcare worker exhaustion and burnout.

\section{Learning and communicating "best" practices without prior evidence}

Daily meetings were held in the evenings, exchanging opinions and sharing experiences. In some, if not all, hospitals, a daily conference was convened to discuss the most difficult cases. The command centre also issued newsletters on a regular basis to all local and external medical teams, providing information including patient management. However, despite the efforts at sharing experiences and regularly updated national guidance, heterogeneity in patient management remained a reality.

The official Wechat account of the Chinese Society of Critical Care Medicine (CSCCM) released updated information daily from the onset of the outbreak in Wuhan. The CSCCM hosted its first webinar on the prevention and management of covid-19 in pregnant women on 24 January 2020, with 36478 attendees from across China. The CSCCM subsequently organised webinars on covid-19-related topics on a daily basis, with microbiologists, virologists, intensivists, infectious diseases specialists, pulmonologists, infection control specialists, and nurses as invited speakers, discussing diagnosis, respiratory support, immunomodulatory therapy, infection control, and so on. Increasingly more time within these webinars was devoted to panel discussions, questions, and answers. By 30 April 2020, the CSCCM had organised 133 webinars on covid-19 with 1329127 participants (fig 1). Though Chinese physicians shared experiences in needed for their patients. To further reassure regular WHO meetings, dissemination of knowledge was mainly through internet platforms (conferencing, social media, journal websites).

\section{Managing the fear factor}

Fully aware that this was a new contagious disease with uncertain transmission characteristics that placed healthcare workers at potential high risk of harm, the command centre dispatched infection control specialists to train every member of the medical rescue teams in infection control policies and measures such as donning and doffing PPE. National experts shared their experiences with newcomers. The focus was on clinical management, although infection control questions were among the most frequently asked.

Some teams were initially reluctant to perform high risk, aerosol-generating procedures (AGPs) and would often over-protect themselves. During their daily rounds, the national experts would encourage clinicians-especially intensive care, emergency department, and anaesthesia staff-to do whatever was staff, the experts would themselves perform procedures such as intubation. In view of experience gained from past major disasters, psychologists were also on hand to support both healthcare workers and patients.

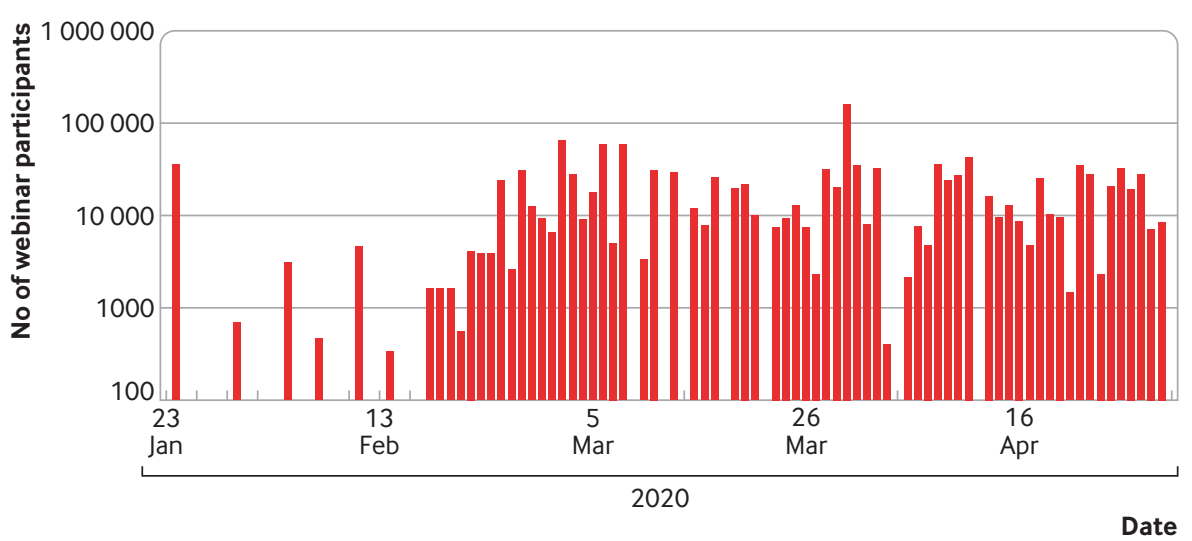

Fig 1 | Daily participants of covid-19 related webinars organised by the Chinese Society of Critical Care Medicine
A further stress was not knowing how this outbreak would progress. The darkest period was from late January to mid-February, when healthcare resources were overwhelmed and the workforce was exhausted. Would it continue to decompensate locally, run rampant across China with even more devastating effects, or evolve into a global pandemic? With the large numbers of covid19 patients requiring admission, the care of non-covid cases was being compromised with delayed admission and greater illness severity on presentation. Optimism increased, however, as more hospitals came on line, external assistance ramped up, equipment, consumables, and PPE became more readily available, and control was regained. Mercifully, from a national perspective, the intensity experienced in Wuhan was not replicated in other big cities, though, tragically, millions have been affected worldwide.

\section{Research efforts}

Research efforts increased over time and enabled early reports published in major international journals to inform on the magnitude of the outbreak and early insights into pathophysiology and management. Initially, there were many observational studies, performed at the discretion of treating physicians, that investigated risk factors as well as specific treatments such as anticoagulation and tocilizumab. ${ }^{67}$ From the haphazard nature of studies at the beginning, a more formalised structure was subsequently established. Some medical rescue teams included fully dedicated researchers with fast-track funding from the Ministry of Science and Technology. Clinical research organisations sent scores of people to facilitate multicentre clinical studies on lopinavir-ritonavir, ${ }^{8}$ remdesivir, ${ }^{9}$ and convalescent plasma. ${ }^{10}$ However, coordination and performance of these studies remained a challenge. 


\section{Lessons learnt}

Failure to prepare is preparing to fail. Having a national emergency plan defines the responsibilities of both central and local government during a pandemic to ensure adequate functioning of the healthcare system; maintaining communication, transportation, public safety, and food supply; and other necessary logistics. The NHC recently requested that designated infectious disease hospitals in every city should be remodelled to comply with all requirements for droplet and airborne precautions, with at least $10 \%$ of hospital beds adequately equipped for management of critically ill patients. With government funding, these hospitals are required to stockpile sufficient equipment and supplies (PPE, equipment, consumables, medications, etc) without relying on external resources for at least the first week of the hospital medical response. In addition, local government has designated back-up hospitals, often tertiary hospitals, in case of patient overload. The issue of Fangcang shelter hospitals has also been discussed in some cities to accommodate patients with no or mild symptoms when most healthcare facilities are overwhelmed. Plans for external assistance have also been prepared, including deployment of medical residents, infectious disease specialists, pulmonologists, intensivists, nurses, and psychology specialists.

Real-time, detailed information is crucial to decision making. Such information should include the number of affected patients, their clinical needs, and healthcare resource status (hospital and intensive care bed availability, staffing levels, and availability of equipment, consumables, and medications). Coordination with epidemiologists and modellers can help to predict the size and trajectory of the patient surge and likely requirements. This approach has been proved both efficient and successful and remains unchanged for subsequent local epidemics.

Early clinical experience does help to understand any novel disease process and identify potentially effective management strategies and treatments. Though necessarily carrying a risk of bias, this serves as a good start, especially when based on an appreciation of the underlying pathophysiology and reasonable deduction. For example, the earlier experience in 2003 with the SARS coronavirus indicated transmission was mainly by droplet and contact. A rational inference could be made that a novel coronavirus would be spread similarly from human to human. Infection control measures should focus accordingly.

Experience sharing should be organised in a systematic, coordinated manner to offer reasonable initial consensus-based guidance on patient management, with modifications as further knowledge becomes available, including randomised trials. Multiple approaches should also be explored for effective dissemination, including, but not limited to, social media, online discussions, newsletters, webinars, and medical journal websites.

The limitations of over-reliance on clinical experience and anecdote should be recognised. Preparations will be put in place to perform appropriate randomised studies to validate observational data. This will include gaining necessary regulatory and ethical approvals in advance to avoid missing the first wave of a pandemic. Multiple research projects have already been proposed to investigate different treatment options targeting viral replication, cytokine storm, and coagulopathy. Because of the unforeseeable scale and nature of any future pandemic, national experts will review these research projects, with priority given to the most important scientific questions. Moreover, blood and respiratory samples should be collected based on a prespecified schedule to facilitate better understanding of pathogenesis and pathophysiology.

This work was supported, in part, by funds from Ministry of Science and Technology of the People's Republic of China (2021YFC0863100).

Contributors and sources: The authors have all been involved in clinical and research aspects of the covid-19 pandemic and response, providing expert advice to international or national health agencies, and commentary through science and social media. $\mathrm{BD}$ and MS conceptualised and drafted this paper. CW took the leading role in organising covid-19-related webinars in China during the pandemic, provided additional ideas and contributed to a series of redrafts. $\mathrm{BD}$ is the guarantor. $\mathrm{BD}$ and $\mathrm{MS}$ contributed equally to the work.

Competing interests: We have read and understood BMJ policy on declaration of interests and have the following interests to declare: BD received funding for research related to covid-19 from Ministry of Science and Technology of the People's Republic of China (2020YFC0841300) and Chinese Academy of Medical Sciences (2020-12M-2-005 and 2019-12M1-001). He sits on advisory boards of National Health Commission of the People's Republic of China for management of covid-19. MS received funding from the Department of Health and Social Care for at-cost development and manufacture of the UCL Ventura CPAP device for use in covid-19 patients. He sits on advisory boards and data and safety monitoring boards for investigational agents for covid-19 disease studied in academic led and industry funded trials.

This article is part of a collection proposed by the Peking University Centre for Public Health and Epidemic Preparedness and Response. Open access fees were funded by individual institutions. The BMJ commissioned, peer reviewed, edited, and made the decision to publish. Li-Ming Li advised on commissioning for this collection. Jin-Ling Tang, Di Wang, and Kamran Abbasi were the lead editors for The BMJ. Bin Du, professor ${ }^{1}$

Chunting Wang, professor ${ }^{2}$

Mervyn Singer, professor ${ }^{3}$

${ }^{1}$ State Key Laboratory of Rare, Complex and Critical Diseases, Medical Intensive Care Unit, Peking Union Medical College Hospital, Beijing 100730, China ${ }^{2}$ Department of Critical Care Medicine, Shandong Provincial Hospital Affiliated to Shandong First Medical University, Jinan, Shandong, China

${ }^{3}$ Bloomsbury Institute of Intensive Care Medicine, Division of Medicine, University College London, London, UK

Correspondence to: B Du

dubin98@gmail.com

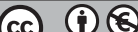

\section{OPEN ACCESS}

This is an Open Access article distributed in accordance with the Creative Commons Attribution Non Commercial (CC BY-NC 4.0) license, which permits others to distribute, remix, adapt, build upon this work non-commercially, and license their derivative works on different terms, provided the original work is properly cited and the use is non-commercial. See: http://creativecommons.org/licenses/by-nc/4.0/.

\section{Check for updates}

Burki T. China's successful control of COVID-19. Lancet Infect Dis 2020;20:1240-1. doi:10.1016/ S1473-3099(20)30800-8.

2 Ksiazek TG, Erdman D, Goldsmith CS, et al, SARS Working Group. A novel coronavirus associated with severe acute respiratory syndrome. N Engl J Med 2003;348:1953-66. doi:10.1056/NEJMoa030781.

3 Chen S, Zhang Z, Yang J, et al. Fangcang shelter hospitals: a novel concept for responding to public health emergencies. Lancet 2020;395:1305-14. doi:10.1016/S0140-6736(20)30744-3.

4 Baidu. National Aid Hubei Medical Team [in Chinese]. https://baike.baidu.com/item/全国援鄂医疗 队/24709605?fr=aladdin

5 Xie J, Tong Z, Guan X, Du B, Qiu H, Slutsky AS. Critical care crisis and some recommendations during the COVID-19 epidemic in China. Intensive Care Med 2020;46:837-40. doi:10.1007/s00134-02005979-7.

6 Zhang Y, Xiao M, Zhang S, et al. Coagulopathy and antiphospholipid antibodies in patients with Covid-19. N Engl J Med 2020;382:e38. doi:10.1056/NEJMc2007575.

7 Xu X, Han M, Li T, et al. Effective treatment of severe COVID-19 patients with tocilizumab. Proc Natl Acad Sci U S A 2020;117:10970-5. doi:10.1073/ pnas.2005615117.

8 Cao B, Wang Y, Wen D, et al. A Trial of LopinavirRitonavir in Adults Hospitalized with Severe Covid-19. N Engl J Med 2020;382:1787-99. doi:10.1056/ NEJMoa2001282.

9 Wang Y, Zhang D, Du G, et al. Remdesivir in adults with severe COVID-19: a randomised, double-blind, placebo-controlled, multicentre trial[Erratum in: Lancet 2020;395:1694]. Lancet 2020;395:1569 78. doi:10.1016/S0140-6736(20)31022-9.

10 Shen C, Wang Z, Zhao F, et al. Treatment of 5 critically ill patients with COVID-19 with convalescent plasma. JAMA 2020;323:1582-9. doi:10.1001/ jama.2020.4783.

Cite this as: $B M J$ 2021;375:e066090 http://dx.doi.org/10.1136/bmj-2021-066090 